(C)2004 IEEE. Personal use of this material is permitted. However, permission to reprint/republish this material for advertising or promotional purposes or for creating new collective works for resale or redistribution to servers or lists, or to reuse any copyrighted component of this work in other works must be obtained from the IEEE. 


\title{
ROBUST LINEAR RECEIVERS FOR SPACE-TIME BLOCK CODED MULTIPLE-ACCESS MIMO WIRELESS SYSTEMS
}

\author{
Yue Rong ${ }^{\dagger} \quad$ Shahram Shahbazpanahi* Alex B. Gershman* \\ ${ }^{\dagger}$ Dept. of Communication Systems, University of Duisburg-Essen, Duisburg, Germany \\ ${ }^{*}$ Dept. of Electrical and Computer Engineering, McMaster University, Hamilton, ON, Canada
}

\begin{abstract}
The problem of joint space-time decoding and interference rejection in multiple-access MIMO wireless communication systems is considered in the case of erroneous or limited channel state information (CSI) at the receiver. Linear beamforming-type techniques that have an improved robustness in such an imperfect CSI case are proposed.
\end{abstract}

\section{INTRODUCTION}

Space-time coding has recently emerged as a powerful approach to exploit spatial diversity and combat fading in MIMO wireless communication systems. Orthogonal space-time block codes (OSTBCs) [1] represent an attractive class of space-time coding techniques because they enjoy full diversity and low decoding complexity. In the point-to-point MIMO communication case, the optimal maximum likelihood (ML) detector for this class of codes consists of a simple linear receiver which maximizes the output signal-to-noise ratio (SNR) and the symbol-by-symbol detector. For each symbol, the ML detector can be interpreted as a matched filter (MF) receiver [2].

In the multiple-access MIMO communication case, the ML receiver has much more complicated structure and prohibitively high complexity as compared with the ML receiver for the pointto-point MIMO case. Therefore, in multiple-access scenarios suboptimal but simple linear receivers can be a good choice.

Several linear receiver techniques have been recently developed for space-time coded multiple access MIMO systems [3]-[6]. Unfortunately, all these techniques assume that the exact CSI is available at the receiver. In practice, this condition can be hardly met because of limited/outdated training as well as the effects of multiple-access interference (MAI) and noise.

In this paper, we develop linear beamforming-type techniques for joint space-time decoding and interference rejection that are based on the worst-case performance optimization and provide an improved robustness in the case of imperfect (erroneous) receiver CSI. Simulation results validate substantial robustness improvements achieved by our techniques.

\section{BACKGROUND}

\subsection{Point-to-Point MIMO}

The relationship between the input and the output of a singleaccess (point-to-point) MIMO system with $N$ transmit and $M$ receive antennas and flat block-fading channel can be expressed as [1]

$$
\mathbf{Y}=\mathbf{X H}+\mathbf{V}
$$

where $\mathbf{Y} \triangleq\left[\begin{array}{lll}\mathbf{y}^{T}(1) & \cdots & \mathbf{y}^{T}(T)\end{array}\right]^{T}, \mathbf{X} \triangleq\left[\mathbf{x}^{T}(1) \cdots \mathbf{x}^{T}(T)\right]^{T}$, and $\mathbf{V} \triangleq\left[\begin{array}{lll}\mathbf{v}^{T}(1) & \cdots & \mathbf{v}^{T}(T)\end{array}\right]^{T}$ are the matrices of the received signals, transmitted signals, and noise, respectively, $\mathbf{H}$ is the $N \times$ $M$ complex channel matrix, $T$ is the block length, and $(\cdot)^{T}$ denotes the transpose. Here, $\mathbf{y}(t)=\left[y_{1}(t) \cdots y_{M}(t)\right], \mathbf{x}(t)=$ $\left[x_{1}(t) \cdots x_{N}(t)\right]$, and $\mathbf{v}(t)=\left[\begin{array}{llll}v_{1}(t) & \cdots & v_{M}(t)\end{array}\right]$ are the complex row vectors of the received signal, transmitted signal, and noise, respectively.

We denote complex information-bearing symbols prior to space-time encoding as $s_{1}, s_{2}, \ldots, s_{K}$ and assume that these symbols belong to (possibly different) constellations $\mathcal{U}_{k}, k=1,2, \ldots, K$. Let $\mathbf{s} \triangleq\left[s_{1} \cdots s_{K}\right]^{T}$. Note that $\mathbf{s} \in \mathcal{S}$ where $\mathcal{S}=\left\{\mathbf{s}^{(1)} \cdots \mathbf{s}^{(L)}\right\}$ is the set of all possible symbol vectors and $L$ is the cardinality of this set. The $T \times N$ matrix $\mathbf{X}(\mathbf{s})$ is called an OSTBC if [1]

- all elements of $\mathbf{X}(\mathbf{s})$ are linear functions of the $K$ complex variables $s_{1}, s_{2}, \ldots, s_{K}$ and their complex conjugates;

- for any arbitrary $\mathbf{s}$, it satisfies

$$
\mathbf{X}^{H}(\mathbf{s}) \mathbf{X}(\mathbf{s})=\|\mathbf{s}\|^{2} \mathbf{I}_{N}
$$

where $\mathbf{I}_{N}$ is the $N \times N$ identity matrix and $\|\cdot\|$ denotes the Euclidean norm of a vector or Frobenius norm of a matrix.

It can be readily verified that the matrix $\mathbf{X}(\mathbf{s})$ can be written as [6]-[8]

$$
\mathbf{X}(\mathbf{s})=\sum_{k=1}^{K}\left(\mathbf{C}_{k} \operatorname{Re}\left\{s_{k}\right\}+\mathbf{D}_{k} \operatorname{Im}\left\{s_{k}\right\}\right)
$$

where $\mathbf{C}_{k} \triangleq \mathbf{X}\left(\mathbf{e}_{k}\right), \mathbf{D}_{k} \triangleq \mathbf{X}\left(j \mathbf{e}_{k}\right), j=\sqrt{-1}$ and $\mathbf{e}_{k}$ is the $K \times 1$ vector having one in the $k$ th position and zeros elsewhere. Using (3), one can rewrite (1) as [6]-[8]

$$
\underline{\mathbf{Y}}=\mathbf{A} \underline{\mathbf{s}}+\underline{\mathbf{V}}
$$

where the "underline" operator for any matrix $\mathbf{P}$ is defined as

$$
\underline{\mathbf{P}} \triangleq\left[\begin{array}{c}
\operatorname{vec}\{\operatorname{Re}(\mathbf{P})\} \\
\operatorname{vec}\{\operatorname{Im}(\mathbf{P})\}
\end{array}\right]
$$

and $\operatorname{vec}\{\cdot\}$ is the vectorization operator stacking all columns of a matrix on top of each other. Here, the $2 M T \times 2 K$ real matrix $\mathbf{A}$ is defined as [6], [8]

$$
\mathbf{A} \triangleq\left[\underline{\mathbf{C}_{1} \mathbf{H}} \cdots \underline{\mathbf{C}_{K} \mathbf{H}} \underline{\mathbf{D}_{1} \mathbf{H}} \cdots \underline{\mathbf{D}_{K} \mathbf{H}}\right]
$$

This matrix has an important property that its columns have the same norms and are orthogonal to each other $\mathbf{A}^{T} \mathbf{A}=\|\mathbf{H}\|^{2} \mathbf{I}_{2 K}$.

In the presence of exact CSI at the receiver, the optimal (ML) space-time decoder uses channel knowledge to find the closest 
point to the received signal in the noise-free observation space $\mathcal{Y}=\left\{\mathbf{Y}^{(1)}, \mathbf{Y}^{(2)}, \ldots, \mathbf{Y}^{(L)}\right\}$, i.e., it obtains [1]

$$
l_{\mathrm{opt}}=\underset{l \in\{1, \ldots, L\}}{\arg \min }\left\|\mathbf{Y}-\mathbf{Y}^{(l)}\right\|
$$

and then uses this index to decode the transmitted bits. Here $\mathbf{Y}^{(l)}$ is the noise-free received signal matrix that corresponds to the vector of information-bearing symbols $\mathbf{s}^{(l)}$.

The ML receiver can also be viewed as a matched filter whose output SNR is maximized [2]. It has been shown in [8] that (7) is equivalent to the MF linear receiver which computes the following estimate of $\underline{\mathbf{s}}$ :

$$
\underline{\hat{\mathbf{s}}}=\frac{1}{\|\mathbf{H}\|^{2}} \mathbf{A}^{T} \underline{\mathbf{Y}}
$$

and builds the estimate of the vector $\mathbf{s}$ as

$$
\hat{\mathbf{s}}=\left[\begin{array}{ll}
\mathbf{I}_{K} & j \mathbf{I}_{K}
\end{array}\right] \underline{\hat{\mathbf{s}}}
$$

The $k$ th element of $\hat{\mathbf{s}}$ is then compared with all points in $\mathcal{U}_{k}$. The closest point is accepted as an estimate of $k$ th entry of $\mathbf{s}$. This procedure is repeated for all $k=1,2, \ldots, K$, that is, the decoding is done symbol-by-symbol.

\subsection{Multiple-Access MIMO}

Let us now consider an uplink multiple-access MIMO communication system shown in Fig. 1. The transmitters are assumed to have the same number of transmitting antennas and to encode the information-bearing symbols using the same OSTBC ${ }^{1}$. The received signal is given by [6]

$$
\mathbf{Y}=\sum_{p=1}^{P} \mathbf{X}_{p} \mathbf{H}_{p}+\mathbf{V}
$$

where $\mathbf{X}_{p}$ is the matrix of transmitted signals of the $p$ th transmitter, $\mathbf{H}_{p}$ is the channel matrix between the $p$ th transmitter and the receiver, and $P$ is the number of transmitters.

Applying the "underline" operator of (5) to (10), we have

$$
\underline{\mathbf{Y}}=\sum_{p=1}^{P} \mathbf{A}_{p} \underline{\mathbf{s}_{p}}+\underline{\mathbf{V}}
$$

where $\mathbf{s}_{p}$ is a $K \times 1$ vector of information-bearing symbols of the $p$ th transmitter and

$$
\mathbf{A}_{p}=\left[\underline{\mathbf{C}_{1} \mathbf{H}_{p}} \cdots \underline{\mathbf{C}_{K} \mathbf{H}_{p}} \underline{\mathbf{D}_{1} \mathbf{H}_{p}} \cdots \underline{\mathbf{D}_{K} \mathbf{H}_{p}}\right] \triangleq\left[\mathbf{a}_{p, 1} \cdots \mathbf{a}_{p, 2 K}\right]
$$

In the multiple-access MIMO case, the MF receiver of (7) becomes highly non-optimal because it ignores the effect of MAI treating it as a noise. In this case, the receiver performance is determined by the signal-to-interference-plus-noise ratio (SINR) rather than the SNR and some cancellation of co-channel interference is required.

Using the array processing-type model (11) and assuming that the first transmitter is the transmitter-of-interest, we can express the output vector of a linear receiver as [6]

$$
\underline{\hat{\mathbf{s}}_{1}}=\mathbf{W}^{T} \underline{\mathbf{Y}}
$$

${ }^{1}$ These assumptions are only needed for notational simplicity and can be relaxed, see [6].

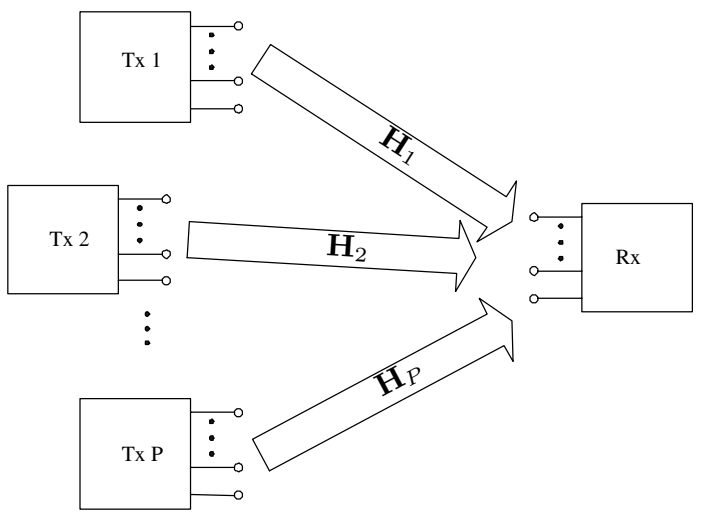

Figure 1: Multiple-access MIMO system.

where $\mathbf{W}=\left[\mathbf{w}_{1} \mathbf{w}_{2} \cdots \mathbf{w}_{2 K}\right]$ is the $2 M T \times 2 K$ real matrix of the receiver coefficients and $\underline{\hat{s}_{1}}$ is the estimate of the vector $\underline{\mathbf{s}}_{1}$ at the receiver output. The vector $\mathbf{w}_{k}$ can be interpreted as the weight vector for the $k$ th entry of $\mathbf{s}_{1}$.

Given the matrix $\mathbf{W}$, the estimate of the vector of information-bearing symbols of the transmitter-of-interest can be computed as $\hat{\mathbf{s}}_{1}=\left[\begin{array}{ll}\mathbf{I}_{K} & j \mathbf{I}_{K}\end{array}\right] \underline{\hat{\mathbf{s}}_{1}}$. Using such a linear estimate, the $k$ th information-bearing symbol can be detected as a point in $\mathcal{U}_{k}$ which is the nearest neighbor to the $k$ th entry of $\hat{\mathbf{s}}_{1}$.

The similarity of the vectorized multiple-access MIMO model (11) and models used in array processing gives an opportunity to design the matrix $\mathbf{W}$ using minimum variance (MV) principle. In particular, in [6] it has been proposed to estimate each entry of $\underline{\mathbf{s}_{1}}$ by minimizing the receiver output power while preserving a unity gain for this particular entry of $\underline{\mathbf{s}_{1}}$, that is,

$$
\min _{\mathbf{w}_{k}} \mathbf{w}_{k}^{T} \hat{\mathbf{R}} \mathbf{w}_{k} \quad \text { s.t. } \mathbf{a}_{1, k}^{T} \mathbf{w}_{k}=1 \text { for all } k=1, \ldots, 2 K
$$

where $\hat{\mathbf{R}}=\frac{1}{Q} \sum_{q=1}^{Q} \underline{\mathbf{Y}_{q}} \underline{\mathbf{Y}}_{q}{ }^{T}$ is the sample estimate of the $2 M T$ $\times 2 M T$ full rank covariance matrix $\mathbf{R} \triangleq \mathrm{E}\left\{\underline{\mathbf{Y}} \underline{\mathbf{Y}}^{T}\right\}$ of the vectorized data (11), $\mathbf{Y}_{q}$ is the $q$ th received data block, $Q$ is the number of data blocks available, and $\mathrm{E}\{\cdot\}$ denotes the statistical expectation.

The solution to (13) is given by [6]

$$
\mathbf{w}_{k}=\frac{1}{\mathbf{a}_{1, k}^{T} \hat{\mathbf{R}}^{-1} \mathbf{a}_{1, k}} \hat{\mathbf{R}}^{-1} \mathbf{a}_{1, k}, \quad k=1, \ldots, 2 K
$$

The form of the obtained MV receiver (14) is similar to that of the minimum variance distortionless response (MVDR) receiver used in beamforming and mimimum output energy (MOE) receiver used in multiuser detection. Although the receiver (14) is able to reject MAI, it does not cancel self-interference [3] which, for each $\mathbf{w}_{k}$, is caused by other entries of $\underline{s}_{1}$ than the $k$ th one. Note that the complete cancellation of self-interference is a very desirable feature because, otherwise, the symbol-by-symbol detector becomes non-optimal [6].

To incorporate the self-interference cancellation feature into (13), it was proposed in [6] to use additional zero-forcing constraints $\mathbf{a}_{1, l}^{T} \mathbf{w}_{k}=0$ for all $l \neq k$. These constraints guarantee that self-interference is completely rejected.

With such additional constraints, the problem (13) can be reformulated as

$$
\min _{\mathbf{W}} \operatorname{tr}\left\{\mathbf{W}^{T} \hat{\mathbf{R}} \mathbf{W}\right\} \quad \text { s.t. } \quad \mathbf{A}_{1}^{T} \mathbf{W}=\mathbf{I}_{2 K}
$$


where $\operatorname{tr}\{\cdot\}$ denotes the trace of a matrix. Using the Lagrange multiplier method, the solution to (15) can be written in the form of the following MV receiver [6]

$$
\mathbf{W}_{\mathrm{MV}}=\hat{\mathbf{R}}^{-1} \mathbf{A}_{1}\left(\mathbf{A}_{1}^{T} \hat{\mathbf{R}}^{-1} \mathbf{A}_{1}\right)^{-1}
$$

To improve the performance in the case of erroneous CSI and sample size, it was proposed in [6] to apply ad hoc diagonal loading to (14) and (16), i.e., to use the matrix $\hat{\mathbf{R}}+\gamma \mathbf{I}_{2 M T}$ instead of $\hat{\mathbf{R}}$ in these receivers. However, it is not clear from [6] what is the optimal choice of $\gamma$ and how it depends on the norm of the CSI errors.

\section{ROBUST LINEAR RECEIVERS}

In this section, we develop new modifications of the techniques (14) and (16) which are robust against imperfect channel knowledge at the receiver. Let us consider the error matrix $\mathbf{H}_{1}-\hat{\mathbf{H}}_{1}$ between the true channel matrix $\mathbf{H}_{1}$ and its presumed (e.g., estimated) value $\hat{\mathbf{H}}_{1}$ and let the Frobenius norm of this error matrix be upper bounded by a known constant $\epsilon$, i.e.,

$$
\left\|\mathbf{H}_{1}-\hat{\mathbf{H}}_{1}\right\| \leq \epsilon
$$

For OSTBCs, it can be proven that

$$
\left\|\mathbf{H}_{1}-\hat{\mathbf{H}}_{1}\right\|=\left\|\mathbf{a}_{1, k}-\hat{\mathbf{a}}_{1, k}\right\|, \quad k=1, \ldots, 2 K
$$

where $\mathbf{a}_{1, k}$ is the $k$ th column of $\mathbf{A}_{1}(\mathbf{H})$ (the true value of the vector $\mathbf{a}_{1, k}$ ) while $\hat{\mathbf{a}}_{1, k}$ is the $k$ th column of the matrix $\mathbf{A}_{1}(\hat{\mathbf{H}})$ (the presumed value $\mathbf{a}_{1, k}$ ). The sought robust modification of (13) should minimize the output power subject to the constraint that the distortionless response is maintained for any estimate $\hat{\mathbf{H}}_{1}$ of the channel matrix $\mathbf{H}_{1}$ that satisfies (17). Using (18), we can write such a robust modification of (13) as

$\min _{\mathbf{w}_{k}} \mathbf{w}_{k}^{T} \hat{\mathbf{R}} \mathbf{w}_{k}$ s.t. $\min _{\left\|\mathbf{e}_{1, k}\right\| \leq \epsilon} \mathbf{w}_{k}^{T}\left(\hat{\mathbf{a}}_{1, k}+\mathbf{e}_{1, k}\right) \geq 1, k=1, \ldots, 2 K$

It can be shown using the results of [9] that the problem (19) is equivalent to

$\min _{\mathbf{w}_{k}} \mathbf{w}_{k}^{T} \hat{\mathbf{R}} \mathbf{w}_{k}$ s.t. $\mathbf{w}_{k}^{T} \hat{\mathbf{a}}_{1, k}-\epsilon\left\|\mathbf{w}_{k}\right\|=1, \quad k=1, \ldots, 2 K$

Applying the Lagrange multiplier method, for each $k=1, \ldots, 2 K$ we obtain the equation

$$
2 \hat{\mathbf{R}} \mathbf{w}_{k}+\mu \epsilon \mathbf{w}_{k} /\left\|\mathbf{w}_{k}\right\|=\mu \hat{\mathbf{a}}_{1, k}
$$

where $\mu$ is the unknown Lagrange multiplier. To get around the problem of computing $\mu$, we assume that a constant modulus constellation is used for which the vector $\mathbf{w}_{k}$ can be arbitrarily rescaled without affecting the performance of a linear receiver. Using this fact and making the rescaling $\mathbf{w}_{k}:=\mathbf{w}_{k} / \mu$, we can rewrite (21) as

$$
\mathbf{w}_{k}=\left(2 \hat{\mathbf{R}}+\left(\epsilon /\left\|\mathbf{w}_{k}\right\|\right) \mathbf{I}_{2 M T}\right)^{-1} \hat{\mathbf{a}}_{1, k}
$$

Note that the term $\epsilon /\left\|\mathbf{w}_{k}\right\|$ can be interpreted as an adaptive diagonal loading factor which is optimally matched to the given level $\epsilon$ of the channel uncertainty. To solve (22), we can apply a similar technique that is developed in [10]. The value of $\left\|\mathbf{w}_{k}\right\|$ can be found as the root of the nonlinear equation

$$
\sum_{i=1}^{2 M T}\left(\frac{\left[\tilde{\mathbf{a}}_{1, k}\right]_{i}}{2 \lambda_{i}\left\|\mathbf{w}_{k}\right\|+\epsilon}\right)^{2}=1
$$

where $\mathbf{U} \boldsymbol{\Lambda} \mathbf{U}^{T}$ is the eigenvalue decomposition of $\hat{\mathbf{R}} ; \boldsymbol{\Lambda}=\operatorname{diag}\{$ $\left.\lambda_{1}, \cdots, \lambda_{2 M T}\right\}$ is the diagonal matrix of eigenvalues of $\hat{\mathbf{R}} ; \tilde{\mathbf{a}}_{1, k}=$ $\mathbf{U}^{T} \hat{\mathbf{a}}_{1, k}$; and $[\cdot]_{i}$ denotes the $i$ th element of a vector.

Inserting the so-obtained $\left\|\mathbf{w}_{k}\right\|$ in the right-hand side of (22), we are able to compute $\mathbf{w}_{k}$. Repeating this procedure for all $k=$ $1, \cdots, 2 K$, we obtain the weight matrix $\mathbf{W}$.

Now, let us develop a robust modification of the receiver (16). To do this, we must add worst-case zero-forcing constraints for self-interference. Following this idea and taking into account that in this case it is impossible to reject self-interference completely, we add to (19) additional constraints to limit the contribution of self-interference to the receiver output power. Then, our problem takes the following form

$$
\begin{aligned}
\min _{\mathbf{w}_{k}} \mathbf{w}_{k}^{T} \hat{\mathbf{R}} \mathbf{w}_{k} \quad \text { s.t. } & \min _{\left\|\mathbf{e}_{1, k}\right\| \leq \epsilon} \mathbf{w}_{k}^{T}\left(\hat{\mathbf{a}}_{1, k}+\mathbf{e}_{1, k}\right) \geq 1 \\
& \max _{\left\|\mathbf{E}_{k}\right\| \leq \eta}\left\|\left(\mathbf{B}_{k}^{T}+\mathbf{E}_{k}^{T}\right) \mathbf{w}_{k}\right\| \leq \delta
\end{aligned}
$$

where

$$
\begin{aligned}
& \mathbf{B}_{k} \triangleq\left[\hat{\mathbf{a}}_{1,1}, \cdots, \hat{\mathbf{a}}_{1, k-1}, \hat{\mathbf{a}}_{1, k+1}, \cdots, \hat{\mathbf{a}}_{1,2 K}\right] \\
& \mathbf{E}_{k} \triangleq\left[\mathbf{e}_{1,1}, \cdots, \mathbf{e}_{1, k-1}, \mathbf{e}_{1, k+1}, \cdots, \mathbf{e}_{1,2 K}\right]
\end{aligned}
$$

$\delta$ is the value which limits the contribution of self-interference, and $\eta$ is the upper bound for $\left\|\mathbf{E}_{k}\right\|$. For OSTBCs, it can be proven that

$$
\eta=\epsilon \sqrt{2 K-1}
$$

Using Cauchy-Schwartz inequality, we can rewrite the constraint (24) as $\left\|\mathbf{B}_{k}^{T} \mathbf{w}_{k}\right\|+\eta\left\|\mathbf{w}_{k}\right\| \leq \delta$. Note that the parameter $\delta$ should be chosen as small as possible (provided that the problem (24) is feasible). However, the issue of feasibility and the choice of $\delta$ can be avoided by treating $\delta$ as a variable to be minimized. Doing so, we obtain the following problem:

$$
\begin{array}{lll}
\min _{\mathbf{w}_{k}, \delta} \mathbf{w}_{k}^{T} \hat{\mathbf{R}} \mathbf{w}_{k}+\delta \quad \text { s.t. } \quad & \mathbf{w}_{k}^{T} \hat{\mathbf{a}}_{1, k}-\epsilon\left\|\mathbf{w}_{k}\right\| \geq 1 \\
& \left\|\mathbf{B}_{k}^{T} \mathbf{w}_{k}\right\|+\eta\left\|\mathbf{w}_{k}\right\| \leq \delta
\end{array}
$$

This is a second-order cone (SOC) programming problem which can be efficiently solved using interior point algorithms [11].

\section{SIMULATIONS}

In simulations, we use the half-rate $(2 K=T=8)$ generalized orthogonal design STBC from [1] and assume $P=2$ transmitters with $N=3$ antennas per transmitter. The receiver has $M=4$ antennas. We assume that the interfering transmitter uses the same OSTBC as the transmitter of interest. Throughout the simulations, the interference-to-noise ratio (INR) is equal to $20 \mathrm{~dB}$ and the QPSK modulation scheme is used. All plots are averaged over 200 independent simulation runs. In each simulation run, the elements of the true channel matrix $\mathbf{H}$ were independently drawn from a complex Gaussian random generator with zero mean and unit variance. Furthermore, in each simulation run, each element of the presumed channel matrix $\hat{\mathbf{H}}$ was generated by drawing a complex Gaussian random variable with zero mean and the variance of 0.2 and adding this variable to a corresponding element of the matrix $\mathbf{H}$. The imperfect CSI case is assumed, i.e., all the receivers tested (except the so-called informed MV receiver) use the presumed channel matrix rather than the true one. The informed MV receiver corresponds to the ideal case of the receiver (16) with 


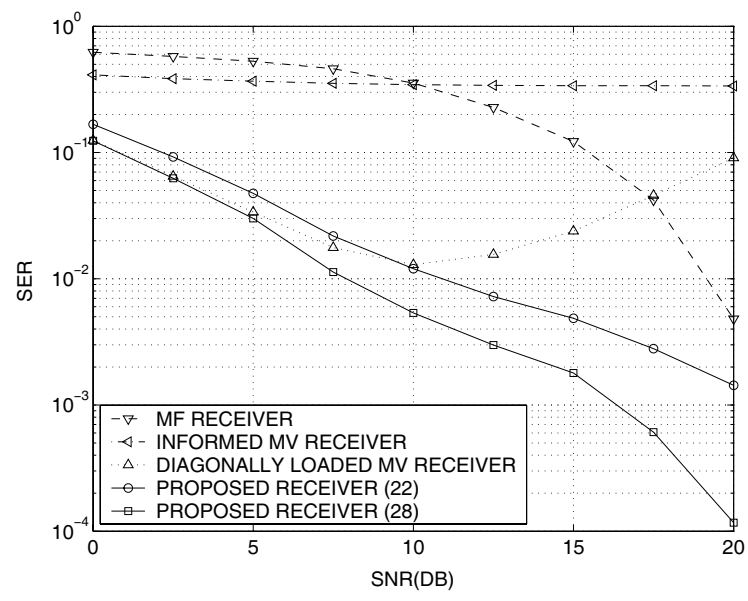

Figure 2: SER versus SNR.

the exactly known $\mathbf{H}$. This receiver does not correspond to any practical situation and is included in our simulations for the sake of comparison only. The proposed robust receivers are compared to the MF receiver (8), the above-mentioned informed MV receiver, and the diagonally loaded MV receiver (which corresponds to the receiver (16) with the sample diagonally loaded covariance matrix $\hat{\mathbf{R}}+\gamma \mathbf{I}_{2 M T}$ ). The diagonal loading factor of $\gamma=10 \sigma^{2}$ is taken where $\sigma^{2}$ is the noise variance. Note that this is a popular ad hoc choice of $\gamma$ that is commonly accepted in the beamforming community.

In Fig. 1, the symbol error rates (SERs) of the linear receivers tested are displayed versus the SNR. In this figure, $Q=100$ data blocks are used to form the data covariance matrix $\hat{\mathbf{R}}$.

Fig. 2 shows the SERs of the receivers tested versus the number of data blocks. In this figure, $\mathrm{SNR}=15 \mathrm{~dB}$.

¿From both figures, it can be seen that the proposed robust receivers greatly outperform all the other receivers tested (including the informed MV receiver). As expected, the receiver (28) substantially outperforms (22) because the former technique takes advantage of an additional nulling of self-interference. The poor performance of the informed MV receiver can be explained by the fact that it is insufficiently robust against finite sample effects. From Fig. 1, we also observe that the performance of the diagonally loaded MV receiver severely degrades at high SNRs. This degradation is due to the fact that this receiver uses the fixed diagonal loading factor.

\section{CONCLUSIONS}

The problem of the design of robust linear receiver techniques for joint space-time decoding and interference rejection in multipleaccess MIMO wireless communication systems has been addressed in the case of an imperfect CSI at the receiver. The proposed robust techniques have been shown to provide substantial performance improvements relative to non-robust linear receivers.

\section{REFERENCES}

[1] V. Tarokh, H. Jafarkhani, and A. R. Calderbank, "Space-time block codes from orthogonal designs," IEEE Trans. Inf. Theory, vol. 45, pp. 1456-1467, July 1999.

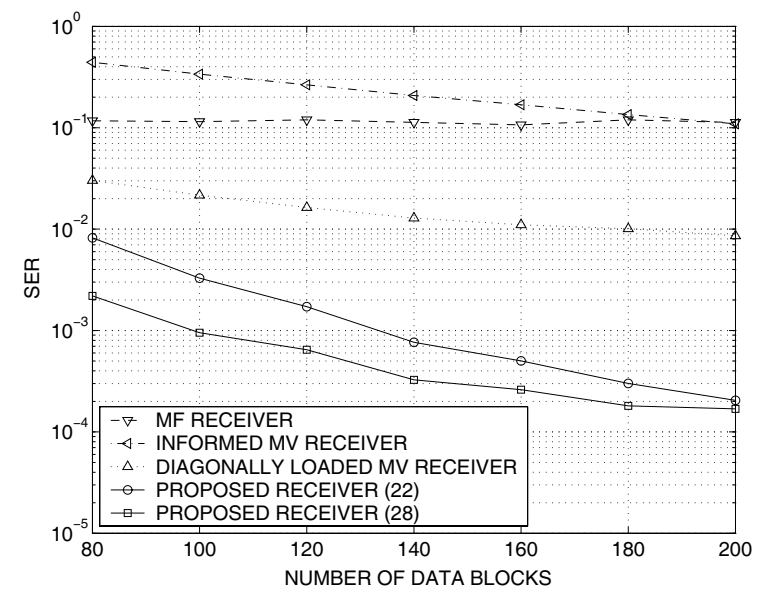

Figure 3: SER versus number of data blocks.

[2] G. Ganesan and P. Stoica, "Space-time block codes: a maximum SNR approach," IEEE Trans. Inf. Theory, vol. 47, pp. 1650-1656, May 2001.

[3] H. Li, X. Lu, and G. B. Giannakis, "Capon multiuser receiver for CDMA systems with space-time coding," IEEE Trans. Signal Processing, vol. 50, pp. 1193-1204, May 2002.

[4] D. Reynolds, X. Wang, and H. V. Poor, "Blind adaptive space-time multiuser detection with multiple transmitter and receiver antennas," IEEE Trans. Signal Processing, vol. 50, pp. 1261-1276, June 2002.

[5] A. F. Naguib, N. Seshadri, and A. R. Calderbank, "Applications of space-time block codes and interference suppression for high capacity and high data rate wireless systems," in Proc. 32nd Asilomar Conf. Signals, Syst., and Comp., Pacific Grove, CA, Nov. 1998, vol. 2, pp. 1803-1810.

[6] S. Shahbazpanahi, M. Beheshti, A. B. Gershman, M. Gharavi-Alkhansari, and K. M. Wong, "Minimum variance linear receivers for multi-access MIMO wireless systems with space-time block coding," IEEE Trans. Signal Processing, submitted (also see Proc. 37 Asilomar Conf. Signals, Syst., and Comp., Pacific Grove, CA, Nov. 2003).

[7] B. Hassibi and B. M. Hochwald, "High-rate codes that are linear in space and time," IEEE Trans. Inf. Theory, vol. 48, pp. 1804-1824, July 2002.

[8] M. Gharavi-Alkhansari and A. B. Gershman, "Constellation space invariance of space-time block codes with application to optimal ML decoding," Proc. IEEE VTC-Fall, Orlando, Florida, USA, Oct. 6-9, 2003.

[9] S. A. Vorobyov, A. B. Gershman, and Z.-Q. Luo, "Robust adaptive beamforming using worst-case performance optimization via second-order cone programming," IEEE Trans. Signal Processing, vol. 51, pp. 313-324, Feb. 2003.

[10] K. Zarifi, S. Shahbazpanahi, A. B. Gershman, and Z.-Q. Luo, "Robust blind multiuser detection based on the worst-case performance optimization of the MMSE receiver," IEEE Trans. Signal Processing, to appear.

[11] J. F. Sturm, "Using SeDuMi 1.02, a matlab toolbox for optimization over symmetric cones," Optimization Methods and Software, vol. 11-12, pp. 625-653, 1999. 